\title{
Mobility Overhead in IEEE 802.15.4e Low Latency Deterministic Networks
}

\author{
A. Darbandi and M. K. Kim ${ }^{1}$ \\ School of Computer and Electrical Engineering, University of Ulsan \\ ${ }^{1}$ mkkim@ulsan.ac.kr
}

\begin{abstract}
IEEE 802.15.4 standard has a limited capacity to ensure low latency for time-critical industrial applications. The IEEE 802.15.4e LLDN mode has been specifically designed for industrial applications requiring low latency and round trip time. One of the main characteristics of LLDN regards the support of device mobility. The mobile devices aiming at association with the PAN coordinator are discovered and configured during discovery and configuration states. These states are isolated from the online state during which transmission of regular readings occurs. As discovery state, configuration state and online state utilize a shared single channel, a long association time at discovery and configuration states leads to a long delay for data transmission in online state, and vice versa. In this paper, we propose a modified superframe structure for fast discovery of mobile devices. Our goal is to minimize association latency for new devices by reducing the latency to enter into the online state. We show the efficiency of our proposed superframe structure compared with the default LLDN via our analysis.
\end{abstract}

Keywords: IEEE 802.15.4e, LLDN, Association delay, Discovery, Configuration

\section{Introduction}

IEEE 802.15.4 [1] standard can be considered as the de-facto physical and MAC infrastructure of the IoT (Internet of Things) paradigm, suitable for low-cost, low-rate, and power constrained wireless devices. However, as it suffers from unbounded delay [4, 5], and no protection against interferences/fading [2, 3, 6], its MAC was amended to support for critical industrial applications. The IEEE 802.15.4e amendment [7] was proposed to support bounded message latency [9, 11, 13], high reliability [8, 10,], scalability [9, 12]. The IEEE 802.15.4e improves the old standard IEEE 802.15.4 by introducing mechanisms such as: Time Slotted Channel Hopping (TSCH), Deterministic and Synchronous Multichannel Extension (DSME), Low Latency Deterministic Network (LLDN).

The DSME mode $[14,15]$ is designed for application domains with deterministic latency constraints but with no low latency requirements, such as home automation, smart building, and entertainment. This protocol proved to be able to achieve low latency (i.e., in the order of hundreds of milliseconds) by using the ultra wide band physical layer, which operates at $850 \mathrm{~kb} / \mathrm{s}$ [14]. However, with the most common IEEE 802.15.4 physical layer operating at $250 \mathrm{~kb} / \mathrm{s}$, low latency is difficult to obtain with DSME [12]. The main reason is due to the fact that, DSME provides a constrained super-frame structure composed of 9 shared timeslots for contention access, and 7 Guaranteed Time Slots (GTS) for the contention free period, where each GTS is exclusively dedicated to a specific source/destination nodes. If more timeslots are needed, an entire super-frame must be added, and this increases latency and round-trip time.

Received (December 17, 2017), Review Result (March 15, 2018), Accepted (March 21, 2018) 
TSCH [11], [15], [21] has been specifically developed for process automation, such as oil and gas industries, chemical products and climate control. It exploits GTS and channel hopping schemes for reliable, deterministic and scalable communication scheme. Each super-frame consists of configurable number of time slots, where $10 \mathrm{~ms}$ length is suggested in the standard. TSCH proved to be not suitable to support applications that require low latency (or round-trip time). The main reason is that, the TSCH uses the classical IEEE 802.15.4 MAC frames, which entail a high overhead, and therefore, a long cycle time [15].

LLDN mode, which is focus of this paper, was introduced to cope with applications requiring low latency and low round-trip time in the order of tens of milliseconds, such as factory automation and control of conveyor belts in cargo and logistics scenarios. As a design target, the network performance should allow for reading sensor data from 20 LLDN devices every $10 \mathrm{~ms}$ [7]. Due to the stringent requirements of low latency and high cyclic determinism, the LLDN mode exploits Time Division Multiple Access (TDMA) medium access strategy within the superframe, a star topology, and a minimum-length superframe structure. Short MAC frames with a one octet MAC header and 2 octets for the Frame Check Sequence (FCS) lead to superframes with durations as short as $10 \mathrm{~ms}$. Star and peer to peer topology defines a network with more than 100 LLDN sensor devices cotrolled by a Personal Area Network (PAN) coordinator, leading to short delivery latencies. Basically, the PAN coordinator is responsible for allocating slots to the individual sensor and actuator nodes. Sensors convey the sensed data to the coordinator, coordinator computes the optimum course of actions and sends the commands to the actuators. A late information delivery may cause loss or severe damage of equipment.

LLDN has three distinct transmission states: Discovery, Configuration and Online. The mobile devices aiming at establishing communication with the coordinator are discovered and configured during Discovery and Configuration states, respectively, after which the network transits into Online state. Data and readings from the devices can only be exchanged during the Online state. In the Online state, LLDN allocates at least one slot to each sensor device, and therefore the length of superframe grows linearly with the number of network nodes. This specification limits the network scalability and raises round trip delay.

Previous works on the LLDN mostly provide methods to improve scalability of Online state and to reduce packet delivery delay [9], [12], [21]. The authors in [9] divided the network into clusters, where each cluster has a different frequency channel to simultaneously operate without any interference with other clusters. [21] studies different configurations of superframe structure in Online state, to describe the relationship and trade-off among superframe length, base timeslot size and data payload with different levels of security. Other works on LLDN [16-18] extend the LLDN star topology to multi-hop networks, by amending the network structure with relay nodes. The relay nodes extend the network coverage area and boost the reliability via retransmitting unsuccessfully delivered packets. In [19], we propose modifications to the LLDN superframe structures, in order to reduce the association latency incurred for new mobile devices which aim to joining the PAN coordinator. Despite the fact that, our proposed approach introduces modifications in LLDN standard, but the results demonstrate that these modifications allow an increase of determinism in Configuration and Discovery states, and it significantly reduces association latency.

Different from the previous works, in this paper we focus on the association delay of mobile devices posed by superframe structure in the Discovery and Configuration states. Our motivation raises from the fact that, transitioning to Discovery and Configuration states prevents the connected devices from transmitting regular readings till completion of both Discovery and Configuration states. A long association time will increase packet delivery delay, while targeted applications of LLDN demand strict requirements in terms 
of latency. In the case of high mobility, where the network has to operate in Discovery and Configuration for a long amount of time, the network performance becomes worse.

The LLDN exploits identical superframe structure at both Discovery and Configuration states. This fact leads to long association delay, as the direction of information exchanges (uplink or downlink communications) at Discovery state is different from that of Configuration state. In order to efficiently associate mobile nodes within a short amount of time, we propose an amendment to the LLDN superframe structure in both Discovery and Configuration states. Our proposed superframe structure reduces the association delay, as it is designed based on direction of information exchanges between mobile device and PAN coordinator, while preserving all communications between PAN coordinator and devices. Our analysis indicates that the latency posed by our proposed superframe structure is more than half of that of the default LLDN.

This paper is organized as follows: Section 2 describes the infrastructure of LLDN. Section 3 describes the problem considered in this paper. Section 4 presents our proposed approach and analyses the association latency overhead, and Section 5 concludes the paper.

\section{LLDN Overview}

The LLDN amendment has been designed to operate in a peer-to-peer star topology, due to the stringent requirements of low-latency applications. All the sensors and actuators exchange data with the PAN coordinator, which acts the centralized controller and drives the medium access schedule for all the sensors and actuators.

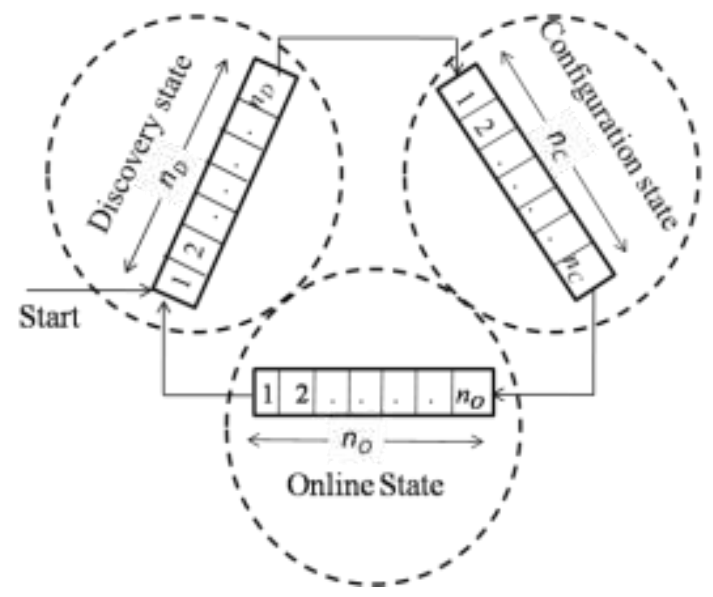

Figure 1. Transitions between Transmission States in LLDN

LLDN divides the time into three states: Discovery, Configuration and Online, see Figure 1. The Discovery state is the first phase during network setup, or it is triggered regularly for the addition of new devices. At this state, the PAN coordinator discovers association requests sent by new mobile devices. In this paper, we assume that the system consists of $M$ new mobile devices willing at association with the PAN coordinator, where $M B=\left\{m_{1}, \ldots, m_{M}\right\}$ represents the set of mobile nodes.

The second phase is the Configuration state, by which the new mobile devices shall receive network configurations. The last state is the Online state, where data and readings from the devices can only be transmitted during this state. The coordinator specifies the state of transmission through periodically transmitted beacons. Each one of the Discovery, Configuration and Online states has a specific superframe structure that is elaborated in the following sections. 


\subsection{Discovery State}

The Discovery state is made up of $N_{D}$ equal-sized Discovery superframes, where each superframe consists of three timeslots, one timeslot for the beacon through which the network is synchronized and the current transmission mode is declared, one timeslot for downlink management and one for uplink management transmissions. The downlink direction is specified for sending data from the PAN coordinator to the LLDN device. The uplink direction is defined for sending data from the LLDN device to the LLDN coordinator. There are only two management timeslots per superframe. Uplink management timeslots are implemented as shared group access timeslots, and the nodes commence transmission based on a slotted CSMA-CA scheme described in [7]. We denote the length of the Discovery superframe by $L_{D}$, the length of beacon timeslot by $T_{B}$, and the length of each management timeslot by $T_{m g t}$. The actual size of each timeslot in octets is presented in [7].

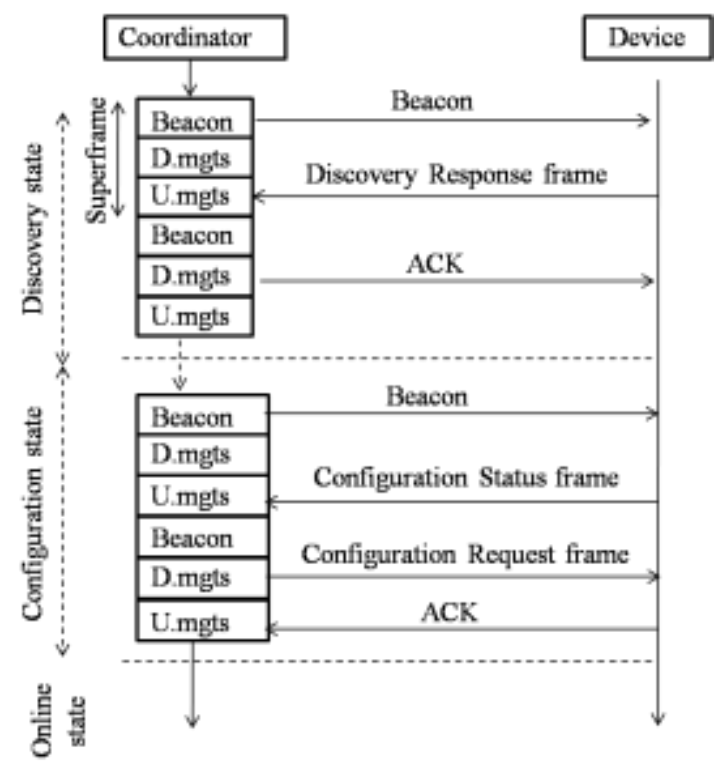

\section{Figure 2. Flow Diagram of LLDN Discovery and Configuration States}

A new device willing to join the network scans for beacons that indicate Discovery state. Once the new device has received a valid beacon, it shall attempt to access the medium in the uplink management timeslot (its time is defined in the beacon), in order to send a Discover Response frame to the LLDN PAN coordinator, see Figure 2. The Discovery Response frame contains the current configuration parameters that have to be transmitted to the PAN coordinator, required for the configuration process at Configuration state. If the coordinator receives the Discovery Response frame correctly, it will reply with an ACK message during the downlink time slot of the next superframe. The new device shall repeat sending the Discover Response frame until it receives an acknowledgment frame for it, or the Discovery state is stopped by the LLDN PAN coordinator.

\subsection{Configuration State}

The Configuration state is made up of $N_{C}$ equal-sized Configuration superframes, where the length of the each superframe is denoted by $L_{C}$. Similar to the Discovery state, in the Configuration state the superframe contains one timeslot for the beacon and two management timeslots for the downlink and uplink transmissions. Here again, uplink 
management timeslots are implemented as shared group timeslots and the medium access is through a slotted CSMA-CA scheme.

Once a node indicates Configuration state through a received beacon, it tries to sends a Configuration Status frame to the LLDN PAN, during an uplink management shared time slot. The Configuration Status frame contains the configuration parameters that are currently configured at the device, such as full MAC address, short MAC address, required timeslot duration and uplink/bidirectional data communications. If LLDN PAN coordinator receives the Configuration Status frame correctly, it would send a Configuration Request frame to the new device, through the downlink frame of next superframe. The configuration parameters in Configuration Request frame include transmission channel, timeslot duration and assigned timeslots to the device. The new device shall repeat sending the Configuration Status frame until it receives a Configuration Request frame for it or the Configuration state is stopped by the LLDN PAN coordinator. After successfully receiving the Configuration Request frame, the device sends an acknowledgment frame to the LLDN PAN coordinator.

\subsection{Online State}

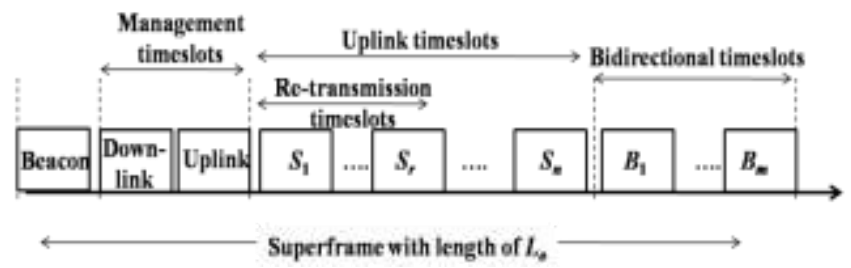

Figure 3. Superframe Structure in Online State

The Online state is made up of $N_{O}$ equal-sized Configuration superframes, where the length of the each superframe is denoted by $L_{O}$. In the Online state, the superframe starts with a beacon which is used for synchronizing devices with the superframe structure. As Figure 3 illustrates, the rest of the LLDN superframe is divided into the following timeslots:

- Two management timeslots, where the first one is downlink and second one is uplink timeslot. These timeslots provide a mechanism for bidirectional transmissions of management data, and their presence is optional in Online state.

- macLLDNnumUplinkTS uplink timeslots for LLDN uplink data transmissions from sensor devices to the PAN coordinator. The default number of timeslots in the uplink is set to 20 and its maximum value is 255 . The first macLLDNnumRetransmitTS timeslots within the uplink section can be reserved for transmission failures occurred in the previous superframe.

Note: In the beacon frame, a Group Acknowledgment field is specified for the PAN coordinator to acknowledge uplink transmissions from previous superframe. The Group Acknowledgment field is a bitmap, where k-th bit corresponds to a LLDN device allocated to $k$-th timeslot after the macLLDNnumRetransmitTS retransmission timeslots. The bit value is one if the corresponding uplink transmission in the previous superframe was successful, and zero otherwise. The Group Acknowledgment field is only present in online mode.

- Bidirectional timeslots are mostly used to exchange control data between PAN coordinator and LLDN actuators. 


\section{Problem Statement}

- The mobile device aiming to associate with a coordinator must scan different channels until it detects a beacon indicating the Discovery state. In the worst case, the new device starts scanning for beacons at the end of Discovery state. Accordingly, this device must scan different channels for a time duration of Scan-Delay ${ }^{\max }=N_{C} L_{C}+N_{O} L_{O}$. This high radio duty cycle leads to increased energy consumption.

- In the Discovery state, the new device must send the Discovery Response frame to the PAN coordinator, through a shared uplink timeslot. As each superframe consists of a single uplink timeslot, the Discovery state must be composed of more than $M$ superframes, where $M$ is the number of new mobile devices.

- In Configuration state, each node must send Configuration Status frame and an Acknowledgment through shared uplink timeslots. As each frame consists of a single uplink timeslot, Configuration state eventually last a long time to be completed.

\section{Modified Superframe Structure}

Our first modification corresponds to the format of beacon frames. Generally, the beacon frame indicates the beginning of a super-frame, synchronizes the devices, contains acknowledgments for the data transmitted in the last superframe and indicates the current transmission mode. We insert additional field of 1 -octet to the MAC payload of LLDN beacon frames, in order to indicate the time interval from the current transmitted beacon frame until the beginning of next Discovery state. This additional field must be inserted in to the beacon frames produced at the Online and Configuration states.

In the worst case, the mobile device starts scanning channels on the end of a beacon slot in the online superframe. Then, by the beacon produced in the next superframe, the mobile device will be notified about the time at which the Discovery state begins. This simple modification has reduced the maximum scanning time from Scan-Delay ${ }^{\max }=N_{C} L_{C}+N_{O} L_{O}$ to the length of a single superframe at Online state, i.e., Scan-Delay ${ }^{\max }=L_{O}$.

The rest of this section describes our proposed superframe structure for the Discovery and Configuration states. The major components of our contribution are as follows. In Section 4.1, we propose our modified superframe structures in the Discovery and Configuration states. In Section 4.3, we present the association latency analysis based on default LLDN superframe structure and our proposed modifications on the superframe structure.

\subsection{Modified Discovery and Configuration Superframes}

Similar to the default LLDN, our proposed superframe of Discovery state is composed of one beacon timeslot and two management timeslots. In order to overcome the long discovery process latency in LLDN, we specify both management timeslots in a superframe for uplink transmissions, i.e., transmission of Discovery Response frames from mobile devices to the PAN coordinator. To acknowledge the transmissions, we take advantage of the group acknowledgement field in the format of beacon frame. In this case, the current beacon frame acknowledges the correct reception of two Discovery Response frames transmitted in the previous superframe, see Figure 4. In addition, we specify an additional 
downlink management timeslot on the end of last superframe, to acknowledge the last transmissions at this superframe.

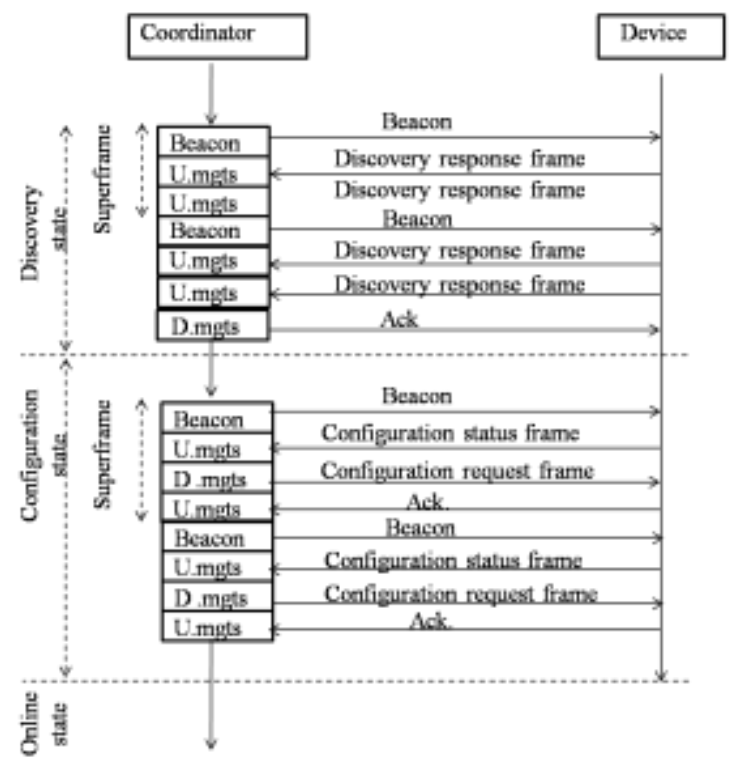

\section{Figure 4. Flow Diagram of our Proposed Discovery and Configuration States}

On the end of Discovery state, the PAN coordinator has the complete knowledge about devices waiting to associate in the Configuration State. We take advantage of this fact to allocate management timeslots exclusively to each device, at Configuration state. Each device can be notified about its assigned management timeslots through some additional parameters included in the beacon frame of Discovery state. This means that, in addition to acknowledging the Discovery Response frame of a device $m_{i}$, the beacon frame of the Discovery state must indicate the assigned slots for $m_{i}$, to send its Configuration Status frame and ACK frame, and to receive its Configuration Request frame. Another approach is that $m_{i}$ finds its allocated slots based the time instant in which $m_{i}$ could achieve access to medium in the Discovery state. When $m_{i}$ is the $k$-th device in the Discovery State that could send its Discovery Response frame, then $m_{i}$ will be the $k$-th device that must be associated to the PAN coordinator in the Configuration state.

Our proposed superframe in the Configuration state is composed of four timeslots. As Figure 4 illustrates, the first timeslot is specified for beacon, and other three timeslots are management timeslots. The first timeslot is uplink management timeslot defined for $m_{i}$ to send Configuration Status frame. The second timeslot is downlink management timeslot specified for PAN coordinator to send Configuration Request frame. The third timeslot is uplink management timeslot allocated for $m_{i}$ to send ACK frame. The main improvement of this approach is its deterministic feature which leads to reduced energy consumption and association latency.

\subsection{Delay Analysis}

In this section, we shall define Discovery Completion Time which is the time interval required for discovering $K$ mobile devices, and Configuration Completion Time which is the time interval required for configuration new mobile devices. Discovery Completion Time for $K$ new mobile devices is the time interval from the beginning of the first Discovery state that serves discovering $K$ new devices until the end of the last slot that sends the last acknowledgment frame from PAN 
coordinator. Similarly, Configuration Completion Time for $K$ mobile devices is the time interval from the beginning of the first Configuration state in the first cycle that serves configuring $K$ new devices until the end of the slot that sends the last acknowledgment frame from a mobile device. We compare the discovering and configuration latencies posed by default LLDN, denoted by $\operatorname{DCT}^{L L D N}(K)$ and $C C T^{L L D N}(K)$, respectively, with our proposed superframe structure, denoted by $D C T(K)$ and $C C T(K)$, respectively.

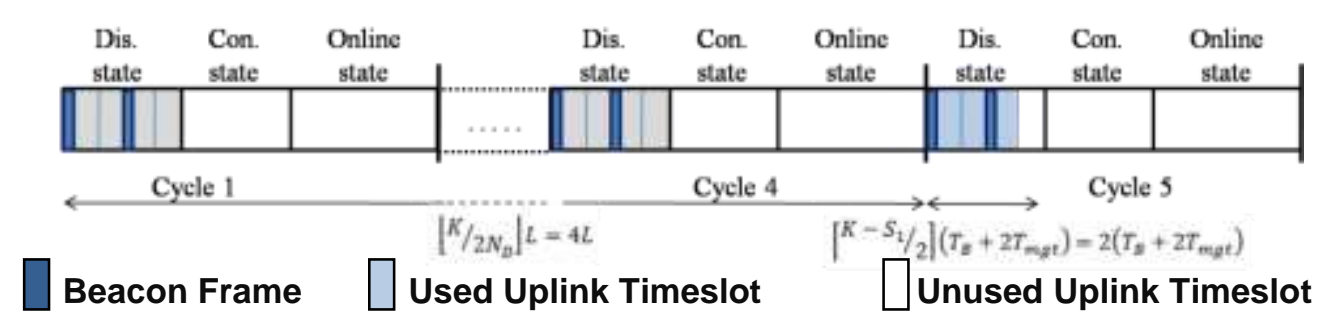

\section{Figure 5. Example Discovery State with $N_{D}=2$ and $K=19$ Mobile Devices waiting to be Discovered. The Discovery States in 4 Cycles are Completely Utilized to Serve 16 New Mobile Devices}

Since at each proposed superframe, two mobile devices can be discovered, totally $\left\lfloor K / 2 N_{D}\right\rfloor L+1$ Discovery states are required. Among these cycles, the Discovery states in the first $\left\lfloor K / 2 N_{D}\right\rfloor$ cycles are fully utilized and the last one is partially utilized, see Figure 5. The time interval from the beginning of the first Discovery state until the end of the last cycle with completely utilized Discovery state is $\left\lfloor K / 2 N_{D}\right\rfloor L$. In the last partially utilized Discovery state, the number of used management timeslots is:

$n_{\text {Dis }}^{\text {last }}=\left\lceil K-S_{1} / 2\right\rceil\left(T_{B}+2 T_{m g t}\right)$

where $S_{1}=\left\lfloor K / 2 N_{D}\right\rfloor 2 N_{D}$ is the number of mobile devices that have been served by previous $\left\lfloor K / 2 N_{D}\right\rfloor$ fully utilized Discovery states. Therefore, $D C T(K)$ can be defined as follow:

$\operatorname{DCT}(K)=\left\lceil K / 2 N_{D}\right\rceil L+\left\lceil K-S_{1} / 2\right\rceil\left(T_{B}+2 T_{m g t}\right)$

To compute $C C T(K)$, we consider two cases. In the first case, all the discovered mobile devices in the current cycle would be configured in the Configuration state of the same cycle. Therefore, the number of superframes in the Configuration state is more than the number of total uplink management timeslots in the Discovery state, i.e., $N_{C} \geq 2 N_{D}$. In the second case, some discovered mobile devices in the current cycle must be configured in the Configuration state of the next cycles. This case occurs due to short duration of Configuration state. In this case, the number of superframes in the Configuration state is less than the number of total uplink management timeslots in the Discovery state, i.e., $N_{C}<2 N_{D}$. 


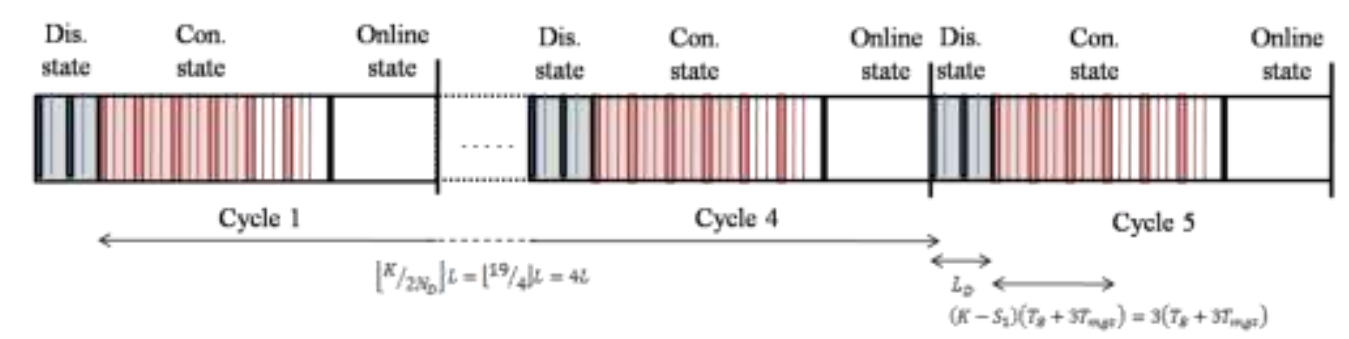

a)

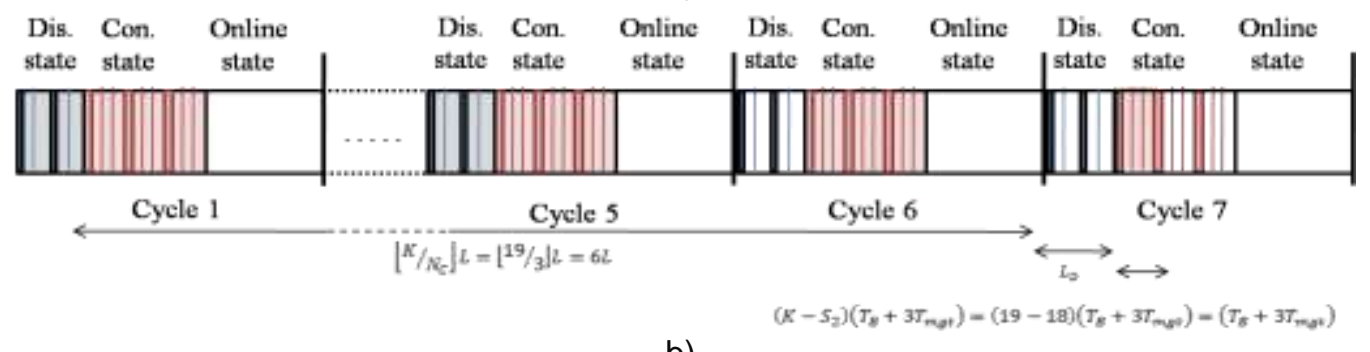

b)

Beacon Frame

Used Timeslot

Unused Timeslot

Figure 6. Example Configuration States. a) $N_{c}=6$ and $K=19$. The Configuration States in First 4 Cycles are Partially Utilized with 4 used Superframes. b) a) $N_{C}=3$ and $K=19$. The Configuration States in First 6 Cycles are Fully Utilized

In the first case, configuration states in the first $\left\lfloor K / 2 N_{D}\right\rfloor$ cycles are partially utilized with $2 N_{D}$ used superframes, and the last one is also partially utilized with $K$ - $S_{1}$ used superframes, see Figure 6-(a). In this case, $C C T(K)$ is:

$C C T(K)_{N_{C} \geq 2 N_{D}}=\left\lceil K / 2 N_{D}\right\rceil L+\left(K-S_{1}\right)\left(T_{B}+3 T_{m g t}\right)+L_{D}$

However when $N_{C}<2 N_{D}$, all the Configuration states in all the cycles from first cycle to cycle $\left\lfloor K / N_{C}\right\rfloor$ are fully utilized, see Figure 6-(b). Therefore, $C C T(K)$ can be defined as follow:

$C C T(K)_{N_{C}<2 N_{D}}=\left\lceil K / N_{C}\right\rceil L+\left(K-S_{2}\right)\left(T_{B}+3 T_{m g t}\right)+L_{D}$

where $S_{2}=\left\lfloor K / N_{C}\right\rfloor N_{C}$ is the number of mobile devices have been served by $\left\lfloor K / N_{C}\right\rfloor$ fully utilized Discovery states.

Now, we shall define Discovery and Configuration Completion Times for associating $K$ mobile devices by the default LLDN. As at each discovery state of default LLDN only one device can be discovered, $D C T^{L L D N}(K)$ can be defined as follow:

$$
D C T^{L L D N}(K)=\left\lceil K / N_{D}\right\rceil L+\left(K-S_{3}\right)\left(T_{B}+2 T_{m g t}\right)
$$

where the first term in the RHS of above equation is the time interval from the beginning of the first LLDN Discovery state until the end of the last LLDN cycle with completely utilized Discovery states, the second term is the number of used management timeslots in 
the last partially utilized Discovery state, and $S_{3}=\left\lfloor K / N_{D}\right\rfloor N_{D}$ is the number of mobile devices that have been served by $\left\lfloor K / N_{D}\right\rfloor$ fully utilized Discovery states.

From Eqs. 2 and 5 it can be seen that given $N_{D}$ Discovery superframes in a Discovery state, the number of cycles to complete discovery procedure in the default LLDN is two times more than that by our proposed superframe structure.

To compute $C C T^{L L D N}(K)$, we consider two cases as studied for computing $C C T^{L L D N}(K)$. In the LLDN Configuration state, each two superframes are specified for the configuration process of a single device. Therefore, when all the discovered mobile devices in the current cycle would be configured in the Configuration state of the same cycle, we have $N_{C} / 2 \geq N_{D}$. When some discovered mobile devices in the current cycle must be configured in the Configuration state of the next cycles, we have $N_{C} / 2<N_{D}$. In the first case, $C C T^{L L D N}(K)$ can be obtained as follow:

$$
C C T(K)_{N_{C} \geq 2 N_{D}}^{L L D N}=\left\lceil K / N_{D}\right\rceil L+\left(K-S_{3}\right)\left(T_{B}+T_{m g t}\right)+L_{D}
$$

However, when $N_{C} / 2<N_{D}, C C T^{L L D N}(K)$ can be defined as follow:

$$
C C T(K)_{N_{C}<2 N_{D}}^{L L D N}=\left\lceil 2 K / N_{D}\right\rceil L+\left(K-S_{4}\right)\left(T_{B}+T_{m g t}\right)+L_{D}
$$

Where $S_{4}=\left\lfloor 2 K / N_{C}\right\rfloor N_{C} / 2$ is the number of mobile devices have been served by $\left\lfloor 2 K / N_{C}\right\rfloor$ fully utilized Discovery states.

From Eqs. 3 and 6, and Eqs. 4 and 7, it can be seen that given $n_{D}$, by LLDN Discovery superframe the required amount of time to discover $M$ mobile devices is two times more than that with our proposed superframe structure. In addition, given $n_{C}$ Configuration superframes in a Configuration state, a Configuration state with our proposed superframe structure can configure same number of mobile devices as in LLDN, but within half amount of time required by LLDN Configuration state. Therefore, the number of cycles in the default LLDN required to complete configuration procedure is two times more than required number of cycles in our approach. This fact gives an obvious observation about effectiveness of our superframe structure. Moreover, the connected devices that are waiting until completion of both Discovery and Configuration states, need to wait for half amount of time to trigger Online state and to start transmitting regular readings.

\section{Conclusion}

This paper has studied the problem of association latency in LLDN networks. We showed that association procedure in LLDN leads to high delay, because the LLDN superframe structure at Discovery and Configuration states does not comply with the direction of information exchanges between mobile device and the PAN coordinator. We proposed a new superframe structure for both Discovery and Configuration states, based on the directions of message exchanges. Our analysis shows that, Discovery and Configuration states with our proposed superframe structures can associate same number of mobile devices as in LLDN, but within a half amount of latency by LLDN. As our feature work, we will adopt our analysis to compute worst case association latency. 


\section{Acknowledgments}

This work was supported by the 2018 Research Fund of University of Ulsan.

This paper is a revised and expanded version of a paper entitled "Reducing Association Latency of Mobile Devices in IEEE 802.15.4e Low Latency Deterministic Networks", presented at the $10^{\text {th }}$ International Conference on Advanced Science and Technology, Ho Chi Minh, Vietnam, February 1-3, 2018 [19].

\section{References}

[1] IEEE Standards Association, "IEEE Standard for Local and Metropolitan Area Networks-Part 15.4: Low-Rate Wireless Personal Area Networks (LR-WPANs), IEEE Standard 802.15.4-2011", (2011), New York, USA.

[2] P. Di Marco, C. Fischione, F. Santucci, and K. H. Johansson, "Modeling IEEE 802.15.4 Networks over Fading Channels", IEEE Transactions on Wireless Communications", vol. 13, no. 10, (2014), pp. 53665381.

[3] E. T. Yazdi, A. Willig, K. Pawlikowski, "Frequency Adaptation for Interference Mitigation in IEEE 802.15.4-based Mobile Body Sensor Networks", Computer Communications, vol 53, no. 1, (2014), pp. $102-119$.

[4] S. Brienza, M. Roveri, D. De Guglielmo, and G. Anastasi, "Just-in-Time Adaptive Algorithm for Optimal Parameter Setting in 802.15.4 WSNs", ACM Transactions on Autonomous and Adaptive Systems, vol. 10, no. 4, (2016), 26 pages.

[5] S. Pollin, M. Ergen, S. Ergen, B. Bougard, L. Van der Perre, I. Moerman, A. Bahai, P. Varaiya, and F. Catthoor, "Performance Analysis of Slotted Carrier Sense IEEE 802.15.4 Medium Access Layer", IEEE Transactions on Wireless Communications, vol. 7, no. 9, (2008), pp. 3359-3371.

[6] G. Anastasi, M. Conti, and M. Di Francesco, "A Comprehensive Analysis of the MAC Unreliability Problem in IEEE 802.15.4 Wireless Sensor Networks", IEEE Transactions on Industrial Informatics, vol. 7, no. 1, (2011), pp. 52-65.

[7] IEEE Standards Association, "IEEE Standard for Local and metropolitan area networks, Part 15.4: LowRate Wireless Personal Area Networks (LR-WPANs) Amendment 1: MAC sublayer, 2012th ed.", (2012), New York, USA.

[8] H. Kapil and C. Siva Ram Murthy, "A Pragmatic Relay Placement Approach in 3-D Space and QLearning-Based Transmission Scheme for Reliable Factory Automation Applications", IEEE Systems Journal, no. 99, (2016), pp. 1-11.

[9] G. Patti; L. Bello, "A Priority-Aware Multichannel Adaptive Framework for the IEEE 802.15.4eLLDN", IEEE Transactions on Industrial Electronics, vol: 63, no. 10, (2016), pp. 6360-6370.

[10] A. Willig; Y. Matusovsky; A. Kind, "Retransmission Scheduling in 802.15.4e LLDN - a Reinforcement Learning Approach with Relayers", 26-th International Telecommunication Networks and Applications Conference (ITNAC), Dunedin, New Zealand, (2016).

[11] M.R. Palattella, N. Accettura, L.A. Grieco, G. Boggia, M. Dohler and T. Engel, "On Optimal Scheduling in Duty-Cycled Industrial IoT Applications using IEEE 802.15.4e TSCH", IEEE Sensors Journal, vol. 13 no. 10, (2013), pp. 3655-3666.

[12] Y. Al-Nidawi, H. Yahya and A. H. Kemp, "Tackling Mobility in Low Latency Deterministic Multihop IEEE 802.15.4e Sensor Network”, IEEE Sensors Journal, vol. 16, no. 5, (2016), pp. 1412-1427.

[13] R. Soua; P. Minet; E. Livolant, "MODESA: An Optimized Multichannel Slot Assignment for Raw Data Convergecast in Wireless Sensor Networks", IEEE 31-st International Performance Computing and Communications Conference (IPCCC), (2012), pp. 91-100

[14] T. Abdelzaher, J. Paso, and J. Iinatti, "Feasibility Study of IEEE 802.15.4e DSME Utilizing IR-UWB and S-Aloha," in Proc. IEEE Int. Symp. Personal, Indoor Mobile Radio Commun., (2013), pp. 18631867, London, U.K.

[15] G. Alderisi, G. Patti, O. Mirabella, and L. Lo Bello, "Simulative Assessments of the IEEE 802.15.4e DSME and TSCH in Realistic Process Automation Scenarios," in Proc. IEEE Int. Conf. Ind. Informat., (2015), pp. 948-955, Cambridge, U.K.

[16] A. Berger, M. Pichler, A. Springer and W. Haslmayr, "Energy Efficient and Reliable Wireless Sensor Networks - An Extension to IEEE 802.15.4e", EURASIP Journal on Wireless Communications and Networking, vol. 2014, no. 126, (2014).

[17] H. Kapil and C. S. R. Murthy, "Rainbow Product Ranking Based Relay Placement and Adaptive Retransmission Scheme for a Reliable 802.15.4e LLDN", In Proceedings of IEEE International Conference on Industrial Technology (ICIT), Seville, Spain, (2015).

[18] G. Patti, G. Alderisi, and L. Lo Bello, "Introducing Multi-level Communication in the IEEE 802.15.4e Protocol: The MultiChannel-LLDN", In Proc. of IEEE Emerging Technology and Factory Automation (ETFA), Barcelona, Spain, (2014). 
[19] A. Darbandi1, S. Lee, M. K. Kim, "Reducing Association Latency of Mobile Devices in IEEE 802.15.4e Low Latency Deterministic Networks", In Proc. Of Advanced Science and Technology Letters (AST), Vietnam, vol. 150, (2018).

[20] L. Dariz and M. Malaguti, G. Ruggeri, "Performance Analysis of IEEE 802.15.4 Real-time Enhancement,” in Proc. IEEE Int. Symp. Ind. Electron., Istanbul, Turkey, (2014), pp. 1475-1480.

[21] M. Anwar, X. Yuanqing, "IEEE 802.15.4e LLDN: Superframe Configuration for Networked Control Systems", Proceedings of the 33rd Chinese Control Conference, Nanjing, China, (2014).

\section{Authors}

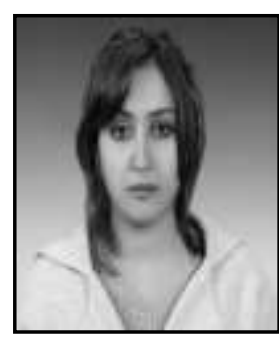

Armaghan Darbandi, received the B.S. degrees in computer engineering from Polytechnic University of Tehran, in 2003; the M.S. and $\mathrm{Ph} . \mathrm{D}$ degree in computer networks from University of Ulsan, South Korea, in 2017.

Currently, she is Research Associate with the University of Ulsan. Her research interests include scheduling real-time communications in networks and real-time systems.

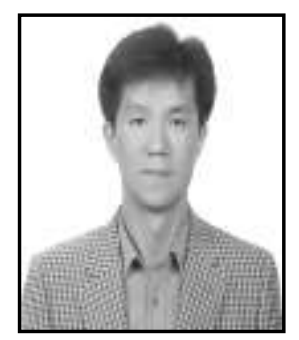

Myung Kyun Kim, received the B.S. degree from Seoul National University of Korea; and the M.S. and Ph.D degrees from KAIST, South Korea.

He is currently a professor in the School of Computer Science and Electrical Engineering, University of Ulsan, South Korea. His research area is real-time communications in wireless networks and industrial communication networks. 